\title{
AN UNDECIDABLE PROPERTY OF DEFINITE INTEGRALS
}

\author{
DANIEL T. STALLWORTH AND FRED W. ROUSH
}

(Communicated by Andreas R. Blass)

\begin{abstract}
We show that it is undecidable whether the definite contour multiple integral of an elementary meromorphic function is zero over an everywhere real analytic manifold on which it is analytic.
\end{abstract}

Previous work on undecidable problems in elementary real analysis has appeared in $[A],[D L]$, and $[R]$. Richardson $[R]$ showed undecidability of some problems concerning a certain class of functions that includes the elementary functions. Those problems include telling if two functions are equal and deciding if a function has an elementary indefinite integral. However he made use of $|x|$ which is algebraic but not smooth. Adler $[\mathrm{A}]$ proved undecidability of whether a root of an equation exists and whether there are solutions to some differential equations. Denef and Lipschitz $[\mathrm{DL}]$ proved undecidability of the existence of certain types of solutions to algebraic differential equations.

Theorem. For any polynomial $P\left(x_{1}, \ldots, x_{n}\right)$ with integer coefficients, consider the integral

$$
\int \cdots \int \frac{\sin (\pi P)}{\pi P} \cot \left(\pi x_{n}\right) \cdots \cot \left(\pi x_{n}\right) e^{-e^{x_{1}+\cdots+x_{n}}} d x_{1} \cdots d x_{n} .
$$

The contour of integration for each variable $x_{i}$ is the arc defined in terms of $u+i v$ by $u=\sec (n \pi v)-1.5$, taking the component closest to the origin, which goes around the nonnegative integers once. To determine, given $P\left(x_{1}, \ldots, x_{n}\right)$, whether this integral is zero is an undecidable problem.

Proof. The integrand is complex analytic.

First we show that the integral converges on the indicated contour because of the exponential factor. Let $M$ be the maximum of the real parts of the $n$ coordinates $x_{i}$. Then $P\left(x_{1}, \ldots, x_{n}\right)$ is bounded by a polynomial in $M$. Therefore $\sin (\pi P)$ grows at a rate which is at most exponential in a polynomial in $M$. In a neighborhood of $P=0, \sin (\pi P) /(\pi P)$ is bounded, and outside this neighborhood $1 / P$ is bounded. Therefore $\left|\frac{\sin (\pi P)}{\pi P}\right|<C e^{C M^{k}}$ for some positive constants $C, k$. The cotangent factors are bounded, since up to periodicity the integration contour stays in a compact set where cotangent is continuous. The expression $e^{\Sigma x_{i}}$ has absolute value determined by the real part of $\sum x_{i}$ and is at least $e^{M-2 n}$ where $n$ is the number of variables. If $u+i v$ is on the contour, then the real part $u$ is at least -2 and the imaginary part $v$ is at most $1 /(2 n)$ in absolute value, up to periodicity, since

Received by the editors February 18, 1994 and, in revised form, January 29, 1996.

1991 Mathematics Subject Classification. Primary 03B25.

Key words and phrases. Undecidability of definite integrals. 
$n \pi v$ must be at most $\pi / 2$. The imaginary part of $\sum x_{i}$ is at most $n(1 / 2 n)$ or $1 / 2$. Therefore

$$
-\operatorname{Re}\left(e^{\Sigma x_{i}}\right)=-e^{\operatorname{Re}\left(\Sigma x_{i}\right)} \cos \left(\operatorname{Im} \sum x_{i}\right) \leq-.5 e^{M-2 n},\left|e^{-e^{\Sigma x_{i}}}\right| \leq e^{-.5 e^{M-2 n}} .
$$

Now the $e^{-e^{\Sigma x_{i}}}$ factor dominates all the others making the product go to zero rapidly. The integral therefore converges.

Next we simplify the integral by rearranging the contour.

The contour for each variable can be broken into separate loops around the poles of order 1 , which occur at nonnegative integer $n$-tuples. We do this one variable at a time, to get a union of tori around the nonnegative integer $n$-tuples as the new domain of integration. The poles in our expression arise from the separate order 1 poles in cotangent where the variables assume integer values. The cotangent function has at 0 a pole of order 1 with residue 1 , so near 0 it looks like $1 / z$ plus a locally holomorphic function. The other poles are translates of this pole by multiples of $\pi$. Then for the torus around each integer $n$-tuple we have an integral which looks locally like a multiple integral of $A /\left(\pi^{n} x_{1} \cdots x_{n}\right)$ around the product of loops in the $x_{i}$ where $A$ is the value of the $\sin (\pi P) /(\pi P)$ and exponential factors at the integer $n$-tuple corresponding to the torus. So the value of the integral around that Cartesian product of loops is $A(2 i)^{n}$. The total integral is $\sum(2 i)^{n} A\left(k_{1}, \ldots, k_{n}\right)$, the sum over all $n$-tuples of nonnegative integers $k_{i}$ of the corresponding values of $A$.

Choose a class of Diophantine equations for which it is undecidable whether they have solutions over $Z$, by the Matijasevitch, Putnam, Davis, Robinson solution of Hilbert's 10th problem. After replacing each variable with a difference of two new variables so that the new variables can be nonnegative, we obtain a class $P$ of Diophantine equations with the additional property that if a solution exists, one exists with all variables positive. The $\sin (\pi P) /(\pi P)$ factor at integers will be 1 if $P$ is 0 and 0 if $P$ is a nonzero integer, since in that case $\sin (\pi P) /(\pi P)$ is the sine of a multiple of $\pi$. Since the exponential factor in $A\left(k_{1}, \ldots, k_{n}\right)$ is positive, $A\left(k_{1}, \ldots, k_{n}\right)$ is positive when $P\left(k_{1}, \ldots, k_{n}\right)=0$ and zero otherwise. Thus the integral in the theorem is nonzero if and only if $P$ vanishes at some nonnegative integers, and the latter is undecidable.

For the class of functions we consider, unlike Richardson's class, equality of functions is decidable by Theorem 5.1 of [DL]. It may be possible to use the constructions of $[\mathrm{DL}]$ to prove a result similar to our theorem. We also conjecture that it is decidable whether or not the integral is zero if the integrand is complex algebraic and the set of which such a contour integral is taken is an immersed closed differentiable manifold.

\section{REFERENCES}

[A] A. Adler, Some recursively unsolvable problems in analysis, Proc. Amer. Math. Soc. 22 (1969), 523-526. MR 40:1277

[DL] J. Denef and L. Lipschitz, Decision problems for differential equations, J. Symbolic Logic 54 (1989), 941-949. MR 91b:03074

[R] D. Richardson, Some undecidable problems involving elementary functions of a real variable, J. Symbolic Logic 33 (1968), 514-520. MR 39:1330

Department of Mathematics, Alabama State University, Montgomery, Alabama 36101-0271

E-mail address: froush@asu.alasu.edu 